Pacific Journal of Mathematic 


\title{
ON REGULAR EXTENSIONS OF MEASURES
}

\author{
George Bachman and Alan Sultan
}

\begin{abstract}
Let $\mathscr{L}$ be a sublattice of $2^{X}$ containing $\varnothing, X$ and let $M R(\mathscr{L})$ be the collection of all bounded nonnegative finitely additive measures defined on $\mathscr{A}(\mathscr{L})$ the algebra generated by $\mathscr{L}$ which are $\mathscr{L}$-regular in the sense that $\mu(E)=$ $\sup \mu(L), L \subset E, L \in \mathscr{L}, E \in \mathscr{A}(\mathscr{L})$. It is shown here that if $\mathscr{L}_{1} \subset \mathscr{L}_{z}$ are sublattices of $2^{x}$ and $\mu \in M R\left(\mathscr{L}_{1}\right)$, then $\mu$ extends to a $\nu \in M R\left(\mathscr{L}_{2}\right)$. Several applications are given.
\end{abstract}

o. Introduction. In previous papers see $[1-4,18,19]$ we were concerned with regular extensions of measures and their applications to several different areas of mathematics. Typically one was given a $\mu \in M R\left(\mathscr{L}_{1}\right)$ and conditions were given for when $\mu$ extended to a $\nu \in M R\left(\mathscr{L}_{2}\right)$ where $\mathscr{L}_{1} \subset \mathscr{L}_{2}$ were sublattices of $2^{x}$. Sufficient conditions for the countable additivity of $\nu$ to follow from that of $\mu$ were also given. In this paper we show that for finitely additive measures, regular extensions always exist. This theorem represents a significant extension of our main theorem of [3] in that now no connection between $\mathscr{L}_{1}$ and $\mathscr{L}_{2}$ is required except that $\mathscr{L}_{1} \subset \mathscr{L}_{2}$. This theorem has a great many applications and some of them are given both to measure extensions and the related concept of measure repleteness, a concept studied in many special cases in [5; 7-10, 14-17].

1* Terminology. Since the results of this paper use to a large extent those of [3], we will adopt the notations and terminology of [3]. The reader need only be familiar with $\S 2$ of that paper. Again we assume that $\varnothing, X$ are elements of all sublattices $\mathscr{L}$ of $2^{X}$ and assume, since this represents no loss of generality, that all measures are bounded and nonnegative. We need the following additional definitions and notations: While $M R(\sigma, \mathscr{L})$ usually denotes the collection of countably additive measures on $\mathscr{L}(\mathscr{L})$, when $\mathscr{L}$ is a delta lattice, we know we may extend any $\mu \in M R(\sigma, \mathscr{L})$ to $\sigma(\mathscr{L})$ retaining the $\mathscr{L}$-regularity. Thus we will assume in the case that $\mathscr{L}$ is a delta lattice that a $\mu \in M R(\sigma, \mathscr{L})$ is already defined on $\sigma(\mathscr{L})$.

If $\mu \in M(\mathscr{L})$, then by the support of $\mu$, denoted by $S(\mu)$, we mean $\cap\{A \in \mathscr{L} \mid \mu(A)=\mu(X)\}$ (even if the measure is defined on $\sigma(\mathscr{L}))$. We say that $\mathscr{L}$ is measure replete if and only if every nonzero $\mu \in M R(\sigma, \mathscr{L})$ has nonempty support.

If $E \subset X$, by $\mathscr{L} \cap E$ we mean $\{L \cap E \mid L \in \mathscr{L}\}$. When we say $E \subset X$ is $\mathscr{L}$-compact, we will mean that the lattice $\mathscr{L} \cap E$ is com- 
pact. When we are working with lattices coming from several different sets we will subscript the lattices appropriately. Thus, $\mathscr{F}_{X}$ will denote the lattice of zero sets of $X$.

If we assume that $X$ and $Y$ are abstract sets and $\mathscr{L}_{1}$ and $\mathscr{L}_{2}$ are sublattices of $2^{X}$ and $2^{Y}$ respectively then $T: X \rightarrow Y$ is called $\mathscr{L}_{1}-\mathscr{L}_{2}$ continuous if $\left\{T^{-1}\left(L_{2}\right) \mid L_{2} \in \mathscr{L}_{2}\right\} \subset \mathscr{L}_{1}$, or more succinctly if $T^{-1}\left(\mathscr{L}_{2}\right) \subset \mathscr{L}_{1} . \quad T$ is called $\mathscr{L}_{1}-\mathscr{L}_{2}$ closed if $T\left(\mathscr{L}_{1}\right) \subset \mathscr{L}_{2}$ where $T\left(\mathscr{L}_{1}\right)=\left\{T\left(L_{1}\right) \mid L_{1} \in \mathscr{L}_{1}\right\}$. If $T$ is a surjection which is $\mathscr{L}_{1}-\mathscr{L}_{2}$ continuous, $\mathscr{L}_{1}-\mathscr{L}_{2}$ closed and such that $T^{-1}\{y\}$ is $\mathscr{L}_{1}$-compact for each $y \in Y$, we call $T \mathscr{L}_{1}-\mathscr{L}_{2}$ perfect or just perfect when the lattices involved are clear. In the case that $X$ and $Y$ are topological spaces and $\mathscr{L}_{1}=\mathscr{F}_{X}$ and $\mathscr{L}_{2}=\mathscr{F}_{Y}$ we get the usual topological definition of perfect map.

2. The general measure extension theorem. We are now ready to prove our main extension theorem. The theorem generalizes substantially our main extension theorem of [3]. We should remark that while the existence of a measure extension in our theorem has been known for some time it is the proof of the existence of an $\mathscr{L}_{2}$-regular measure which is new.

Before presenting the main extension theorem we introduce some of the notations used in the proof. If $\mu \in M R\left(\mathscr{L}_{1}\right)$ then $\mu_{*}$, the (finitely additive) inner measure associated with $\mu$, and $\mu^{*}$, the (finitely additive) outer measure associated with $\mu$ are defined as follows: For any $E \in 2^{X}, \mu_{*}(E)=\sup \mu(B), B \in \mathscr{A}\left(\mathscr{L}_{1}\right), B \subset E, \mu^{*}(E)=$ inf $\mu(B), B \in \mathscr{L}\left(\mathscr{L}_{1}\right), B \supset E$. If $\mathscr{L}_{1}$ and $\mathscr{L}_{2}$ are sublattices of $2^{X}$ and $\mathscr{B}$ is a subalgebra of $2^{x}$ such that $\mathscr{A}\left(\mathscr{L}_{1}\right) \subset \mathscr{B} \subset \mathscr{A}\left(\mathscr{L}_{2}\right)$, then if $\mu \in M(\mathscr{B})$ we say $\mu$ is $\mathscr{L}_{2}$ regular if $\mu(B)=\sup \mu\left(L_{2}\right), L_{2} \in \mathscr{L}_{2} \cap \mathscr{B}$ and $L_{2} \subset B$. We can now present our general measure extension theorem.

THeOREM 2.1. Suppose $\mathscr{L}_{1} \subset \mathscr{L}_{2}$ are sublattices of $2^{x}$. Then if $\mu \in M R\left(\mathscr{L}_{1}\right), \mu$ extends to a $\nu \in M R\left(\mathscr{L}_{2}\right)$. The extension is unique if $\mathscr{L}_{1}$ separates $\mathscr{L}_{2}$. If $\mathscr{L}_{2}$ is $\mathscr{L}_{1}$ countably paracompact, or $\mathscr{L}_{1}$ c.b. or $\mathscr{L}\left(\mathscr{L}_{1}\right)$ countably paracompact $\left(=\mathscr{A}\left(\mathscr{L}_{1}\right)\right.$ c.b.) then if $\mu \epsilon$ $M R\left(\sigma, \mathscr{L}_{1}\right), \nu \in M R\left(\sigma, \mathscr{L}_{2}\right)$.

Proof. Consider the collection of ordered pairs $\left(\mathscr{A}_{\alpha}, \mu_{\alpha}\right)$ where $\mathscr{A}\left(\mathscr{L}_{1}\right) \subset \mathscr{A}_{\alpha} \subset \mathscr{A}\left(\mathscr{L}_{2}\right)$ and $\mu_{\alpha}$ is an $\mathscr{L}_{2}$-regular measure defined on $\mathscr{A}_{\alpha}$ which extends $\mu$. Partially order these pairs as follows: $\left(\mathscr{A}_{\alpha}, \mu_{\alpha}\right) \leqq\left(\mathscr{A}_{\beta}, \mu_{\beta}\right)$ if and only if $\mathscr{A}_{\alpha} \subset \mathscr{A}_{\beta}$ and $\mu_{\beta}$ extends $\mu_{\alpha}$. Then $\left\{\left(\mathscr{A}_{\alpha}, \mu_{\alpha}\right)\right\}$ is trivially inductively ordered and hence by Zorn's lemma, there is a maximal element $(\mathscr{B}, \nu)$. We will show that $\mathscr{B}=\mathscr{H}\left(\mathscr{L}_{2}\right)$ and that $\nu$ is the required measure. 
First, suppose that there is an $F \in \mathscr{L}_{2}$ such that $F \notin \mathscr{B}$. To show this is impossible. Consider $\mathscr{B}_{F}=$ the algebra generated by $\mathscr{B}$ and $F$. It is well known and easy to show that $\mathscr{B}_{F}$ consists of all sets $E$ of the form $E=(A \cap F) \cup\left(B \cap F^{\prime}\right)$ where $A, B \in \mathscr{B}$. Define a set function $\nu_{F}$ on $\mathscr{B}_{F}$ as follows: for any $E \in \mathscr{B}_{F}, \nu_{F}(E)=$ $\nu^{*}(E \cap F)+\nu_{*}\left(E \cap F^{\prime}\right)$. We will show that $\nu_{F}$ is an $\mathscr{L}_{2}$-regular measure defined on $\mathscr{B}_{F} \supsetneq \mathscr{B}$ and that $\nu_{F}$ extends $\nu$. This will contradict the maximality of $\nu$ and prove that $F \in \mathscr{B}_{2}$, for all $F \in \mathscr{L}_{2}$. It will then follow that $\mathscr{A}\left(\mathscr{L}_{2}\right) \subset \mathscr{B}$ since $\mathscr{B}$ is an algebra, and since $\mathscr{B} \subset \mathscr{A}\left(\mathscr{L}_{2}\right)$ to begin with, we will get that $\mathscr{B}=\mathscr{A}\left(\mathscr{L}_{2}\right)$.

The proof that $\nu^{*}$ and $\nu_{*}$ are additive on sets of the form $A \cap F$ and $B \cap F^{\prime}$ respectively where $A, B \in \mathscr{B}$ is simple and follows as in [11], page 269, Lemma. The fact that $\nu_{F}$ is a measure on $\mathscr{B}_{F}$ and $\nu_{F}$ extends $\nu$ is simple and again follows from Theorem 1 of [11], pg. 269. We need only prove that $\nu_{F}$ is $\mathscr{L}_{2}$-regular.

Suppose then $E \in \mathscr{B}_{F}$. Then $E=(A \cap F) \cup\left(B \cap F^{\prime}\right)$ for some $A, B \in \mathscr{B}$. Thus $E \cap F=A \cap F$ and $E \cap F^{\prime}=B \cap F^{\prime} . \nu_{*}\left(E \cap F^{\prime}\right)=\nu_{*}$ $\left(B \cap F^{\prime}\right)=\sup \left\{\nu(C) \mid C \subset B \cap F^{\prime}, C \in \mathscr{B}\right\}=\sup \left\{\nu\left(L_{2}\right) \mid L_{2} \in \mathscr{B} \cap \mathscr{L}_{2}, L_{2} \subset\right.$ $\left.B \cap F^{\prime}\right\}$. Thus there is an $L_{2} \in \mathscr{B} \cap \mathscr{L}_{2}$ such that $L_{2} \subset B \cap F^{\prime}$ and such that $\nu_{*}\left(B \cap F^{\prime}\right)<\nu_{*}\left(L_{2}\right)+\varepsilon / 2 \leqq \nu_{*}\left(L_{2} \cap F^{\prime}\right)+\varepsilon / 2$.

Consider now $\nu^{*}(E \cap F)=\nu^{*}(A \cap F)$. There is an $L_{2} \in \mathscr{L}_{2} \cap \mathscr{B}$, $L_{2} \cdot \subset A$ such that $\nu\left(A-\hat{L_{2}}\right)<\varepsilon / 2$, since $\nu$ is $\mathscr{L}_{2}$ regular. It follows that

$$
\begin{aligned}
\nu^{*}\left\{(A \cap F)-\left(L_{2}^{\hat{\imath}} \cap F\right)\right\} & =\nu^{*}\left(A \cap F-L_{2}^{\hat{}}\right) \\
& \leqq \nu^{*}\left(A-L_{2}\right)=\nu\left(A-L_{2}^{\hat{}}\right)<\varepsilon / 2 .
\end{aligned}
$$

Since $\nu^{*}$ is a measure on sets of the form $A \cap F$ where $A \in \mathscr{B}$ (again [11], pg. 269, Lemma) we have:

$$
\nu^{*}\left(A \cap F-\hat{L_{2}} \cap F\right)=\nu^{*}(A \cap F)-\nu^{*}\left(L_{2} \cap F\right)<\varepsilon / 2 .
$$

Let $L_{2}^{*}=\left(\hat{L_{2}} \cap F\right) \cup L_{2} \in \mathscr{C}_{2} \cap \mathscr{B}_{F^{*}}$. Then $\nu_{F^{\prime}}\left(\left(\hat{L_{2}} \cap F\right) \cup L_{2}\right)=\nu^{*}\left(\hat{L_{2}} \cap\right.$ $F)+\nu_{*}\left(L_{2} \cap F^{\prime}\right)$ and therefore

$$
\begin{aligned}
\nu_{H^{\prime}}(E)-\nu_{F}\left(L_{2}^{*}\right)= & \nu_{F}(E)-\nu_{F}\left(\left(L_{2} \cap F\right) \cup L_{2}\right) \\
= & \nu^{*}(A \cap F)-\nu^{*}\left(L_{2} \cap F\right)+\nu_{*}\left(B \cap F^{\prime}\right)-\nu_{*}\left(L_{2} \cap F^{\prime}\right) \\
& <\varepsilon / 2+\varepsilon / 2=\varepsilon .
\end{aligned}
$$

Thus $\nu_{F}$ is $\mathscr{L}_{2}$ regular. Since this contradicts the maximality of $\nu, \mathscr{P}_{2} \subset \mathscr{B}$ and $\nu$ is the required measure.

To prove that $\nu$ is $\sigma$-smooth if $\mu$ is, and $\mathscr{L}_{2}$ is $\mathscr{L}_{1}$-countably paracompact, suppose $L_{2, n} \downarrow \varnothing, n=1,2,3, \cdots$, where $L_{2, n} \in \mathscr{L}_{2}$. Then by hypothesis there exist $L_{1, n} \in \mathscr{L}_{1}$ such that $L_{2, n} \subset L_{1, n}^{\prime} \varnothing$. Thus $\nu\left(L_{2, n}\right) \subset \nu\left(L_{1, n}^{\prime}\right)=\mu\left(L_{1, n}^{\prime}\right) \downarrow 0$. Thus $\nu$ is $\sigma$-smooth on $\mathscr{L}_{2}$ and since $\nu$ 
is $\mathscr{L}_{2}$-regular $\nu$ is $\sigma$-smooth on $\mathscr{L}\left(\mathscr{L}_{2}\right)$. The proof that $\nu \in M R\left(\sigma, \mathscr{P}_{2}\right)$ is $\mathscr{L}_{2}$ is $\mathscr{A}\left(\mathscr{L}_{1}\right)$ c.b. is analogous. Finally to prove that if $\mathscr{L}_{1}$ separates $\mathscr{L}_{2}$ every $\mu \in M R\left(\mathscr{L}_{1}\right)$ extends uniquely to a $\nu \in M R\left(\mathscr{L}_{2}\right)$ we refer the reader to the proof of Theorem 4.2, pg. 303 of [3].

REMARK 2.2. If $\mathscr{L}_{2}$ is countably compact and if $\mu \in M R\left(\sigma, \mathscr{L}_{1}\right)$, then $\nu \in M R\left(\sigma, \mathscr{L}_{2}\right)$ regardless. This follows from Theorem 2.1, pg. 295 of [3].

This remark gives us the first corollary which generalizes the main extension theorem of Hardy and Lacey [6].

COROLlaRY 2.3. If $X$ is a topological space which has two topologies $\mathscr{T}_{1}$ and $\mathscr{F}_{2}$ with closed sets $\mathscr{F}_{1}$ and $\mathscr{F}_{2}$ and if $\mathscr{F}_{2}$ is countably compact, then every $\mu \in M R\left(\sigma, \mathscr{F}_{1}\right)$ extends to a $\nu \in$ $\operatorname{MR}\left(\sigma, \mathscr{F}_{2}\right)$.

The next corollary gives us a well known theorem of Marik (see [13]).

COROLlaRY 2.4. If $X$ is a countably paracompact and normal space, then every $\mu \in M R(\sigma, \mathscr{P})$ extends to a $\nu \in M R(\sigma, \mathscr{F})$. The extension here is unique.

Proof. In this case $\mathscr{F}_{X}$ is $\mathscr{Z}_{X}$ c.b. as is easy to see from the normality of the space and the fact that it is countably paracompact.

We also have the following:

Corollary 2.5. If $X$ is a Tychonoff space such that $\mathscr{F}_{X}$ is $\mathscr{\mathscr { N }}_{X}$ countably paracompact, then every $\mu \in M R\left(\sigma, \mathscr{F}_{x}\right)$ extends to a $\nu \in$ $\operatorname{MR}\left(\sigma, \mathscr{F}_{x}\right)$.

It is natural to ask whether there is any relation between the conditions $\mathscr{F}_{X}$ is $\mathscr{Z}_{X}$ countably paracompact, and $\mathscr{F}_{X}$ is countably paracompact (i.e., $X$ is countably paracompact in the usual topological sense). Without normality the answer is no. Indeed in the Tychonoff plank $\mathscr{F}_{X}$ is $\mathscr{K}_{X}$ countably paracompact but it is not countably paracompact. In addition Mack and Johnson [12], pg. 240-241 have constructed an example which is countably paracompact but where $\mathscr{F}_{x}$ is not $\mathscr{F}_{x}$ countably paracompact.

Obviously the following well known extension theorem is a trivial corollary of the above theorem. 
CoRollaRy 2.6. If $\mathscr{A}_{1} \subset \mathscr{A}_{2}$ are subalgebras of $2^{x}$, every $\mu \epsilon$ $M\left(\mathscr{A}_{1}\right)$ extends to $a \nu \in M\left(\mathscr{A}_{2}\right)$.

Proof. $M\left(\mathscr{A}_{1}\right)=M R\left(\mathscr{A}_{1}\right), M\left(\mathscr{A}_{2}\right)=M R\left(\mathscr{A}_{2}\right)$ since $\mathscr{A}_{1}$ and $\mathscr{A}_{2}$ are complemented.

We should mention that our extension theorem given in [3] has some advantages over this: It gives us all the $\mathscr{L}_{2}$ regular extension of $\mu$ under those circumstances. Indeed it shows that all linear functionals extending the one associated with $\mu$ in the natural way gives rise to an extension of $\mu$. However, in applications all one needs most of the time is the existence of some $\mathscr{L}_{2}$-regular measure, and that's where the theorem presented here is more useful since the assumptions here are minimal.

Other corollaries follow simply in view of the generality of the theorem and so we move on and mention some applications to direct images of measure replete spaces.

THEOREM 2.7. Let $\mathscr{L}_{1}$ and $\mathscr{L}_{3}$ be delta lattices of subsets of $X$ with $\mathscr{L}_{1} \subset \mathscr{L}_{3} \subset \tau\left(\mathscr{L}_{1}\right)$ and either $\sigma\left(\mathscr{L}_{1}\right) \subset s\left(\mathscr{L}_{1}\right)$ or $\mathscr{L}_{1}$ semiseparates $\mathscr{L}_{3}$. Let $\mathscr{L}_{2} \subset \mathscr{L}_{4}$ be delta lattices of subsets of $Y$ such that $\mathscr{L}_{4}$ is $\mathscr{L}_{2}$ countably paracompact. Then if $T$ is an $\mathscr{L}_{3}-\mathscr{L}_{4}$ continuous surjection such that $\mathscr{L}_{3}$ is $T^{-1}\left(\mathscr{L}_{4}\right)$ countably paracompact then $\mathscr{L}_{1}$ measure replete implies $\mathscr{L}_{2}$-measure replete.

Proof. If $\mu \in M R\left(\sigma, \mathscr{L}_{3}\right)$ and $\mu_{1}=\mu_{\left(\mathscr{L}_{1}\right)}$, then by Theorem 2.3 of [3] we see that $\mu_{1} \in M R\left(\sigma, \mathscr{L}_{1}\right)$. Thus $S\left(\mu_{1}\right) \neq \varnothing$. Since $S(\mu)=$ $S\left(\mu_{1}\right), \mathscr{L}_{3}$ is measure replete. Now let $\nu \in M R\left(\sigma, \mathscr{L}_{2}\right)$. By Theorem $2.1, \nu$ extends to a $\nu_{1} \in M R\left(\sigma, \mathscr{L}_{4}\right)$. Define $\rho_{1}$ on $\sigma\left(T^{-1}\left(\mathscr{L}_{4}\right)\right)=$ $T^{-1}\left(\sigma\left(\mathscr{L}_{4}\right)\right)$ by $\rho_{1} T^{-1}(B)=\nu_{1}(B)$. Then $\rho_{1} \in M R\left(\sigma, T^{-1}\left(\mathscr{L}_{4}\right)\right)$. Since $T$ is an $\mathscr{L}_{3}-\mathscr{L}_{4}$ continuous surjection it is easy to see that $\rho_{1}$ is well defined and thus again by Theorem $2.1 \rho_{1}$ extends to a $\rho \in M R(\sigma$, $\left.\mathscr{L}_{3}\right)$. As noted above $\mathscr{L}_{3}$ is measure replete and thus $S(\rho) \neq \varnothing$. Since $\nu_{1}=\rho T^{-1}, S\left(\nu_{1}\right) \neq \varnothing$, hence $S(\nu) \neq \varnothing$ since $S(\nu) \supset S\left(\nu_{1}\right)$. Since $\nu$ was an arbitrary element of $M R\left(\sigma, \mathscr{L}_{2}\right)$ we see that $\mathscr{L}_{2}$ is measure replete.

As a typical type corollary one has

CoROLlaRY 2.8. Let $X$ be a Tychonoff space and $T$ be a perfect map from $X$ onto a space $Y$ such that $\mathscr{F}_{Y}$ is $\mathscr{Z}_{Y}$ countably paracompact (or c.b.). Then $\mathscr{\varkappa}_{X}$ is measure replete implies $\mathscr{Z}_{Y}$ is measure replete. 
Proof. In the theorem take $\mathscr{L}_{1}=\mathscr{Z}_{X}, \mathscr{L}_{2}=\mathscr{Z}_{Y}, \mathscr{L}_{3}=\mathscr{F}_{X}$, $\mathscr{L}_{4}=\mathscr{F}_{Y} . \quad$ As is well known $\sigma\left(\mathscr{F}_{X}\right) \subset S\left(\mathscr{Z}_{X}\right)$, and $\mathscr{L}_{1} \subset \mathscr{L}_{3} \subset \tau\left(\mathscr{L}_{1}\right)$ since in a Tychonoff space $\mathscr{F}_{X}$ forms a base for $\mathscr{F}_{x}$. To see that $\mathscr{L}_{3}$ is $T^{-1}\left(\mathscr{L}_{4}\right)$ countably paracompact (or c.b.) suppose $F_{n} \downarrow \varnothing$ where $F_{n} \in \mathscr{F}_{X}$. Then by Lemma 5.2, pg. 309 of [3], $T\left(F_{n}\right) \downarrow \varnothing$. But $T\left(F_{n}\right) \subset Z_{n}^{\prime}$ (or $Z_{n}$ ) for $Z_{n} \in \mathscr{Z}_{Y}$. Thus by taking preimages we get the result.

CoRollary 2.9. If $T: X \rightarrow Y$ is an open perfect map where $X$ and $Y$ are Tychonoff spaces, then $\mathscr{Z}_{X}$ measure replete implies $\mathscr{F}_{Y}$ is measure replete.

Proof. In the theorem take $\mathscr{L}_{1}=\mathscr{L}_{3}=\mathscr{Z}_{X}$; and $\mathscr{L}_{2}=\mathscr{L}_{4}=\mathscr{Z}_{Y}$ and note that for open perfect mappings, $T\left(\mathscr{F}_{X}\right) \subset \mathscr{Z}_{Y}$ [20] Corollary 15.14). By Lemma 5.3, pg. 312 of [3] $\mathscr{L}_{3}$ is $T^{-1}\left(\mathscr{L}_{4}\right)$ countably paracompact.

REMARK. Theorem 2.1 can be strengthened if we require $\mathscr{L}_{1}$ and $\mathscr{L}_{2}$ are closed under countable intersections. More precisely, if $\mathscr{L}_{2}$ is $\sigma\left(\mathscr{L}_{1}\right)$ countably paracompact in this case then if $\mu$ is countably additive, so is the extension $\nu$.

ACKNOWLEDGMent. We would like to thank the referee for pointing out to us some examples and counter-examples which we were unaware of and for pointing out to us a way shortening considerably our original proof of Theorem 2.1.

\section{REFERENCES}

1. G. Bachman and A. Sultan, Extensions of regular lattice measures with topological applications, J. Math. Anal. Appl., 57 No. 3, (1977), 539-559.

2. - Measure theoretic techniques in topology and mapping of replete and measure replete spaces, Bull. Austr. Math. Soc., 18 No. 2, (1978), 267-285.

3. — Regular lattice measures: Mappings and spaces, Pacific J. Math., 68 No. 2, (1976), 291-321.

4. - Representation of linear functionals on spaces of continuous functions, Repletions and General Measure Extensions, J. Math. Anal. Appl., 67 No. 2, (1979), 277-293.

5. R. J. Gardner, The regularity of Borel measures and Borel measure compactness, Proc. London Math. Soc., 330 (1975), 95-113.

6. J. Hardy and H. Lacey, Extensions of regular Borel measures, Pacific J. Math., 24 (1968), 271-282.

7. R. Haydon, On compactness in spaces of measures and measure compact spaces, Proc. London Math. Soc.. (3) 29, (1974), 1-16.

8. R. B. Kirk, Locally compact and B-compact spaces, Neder, Acad. Wetensch. Proc. Ser., A72 (1969), 333-344.

9. - Measures on topological spaces and B-compactness, Neder. Acad. Wetensch. 
Proc. Ser., A72 (1969), 172-183.

10. J. D. Knowles, Measures on topological spaces, Proc. London Math. Soc., 17 (1967), 139-156.

11. J. Los and E. Marczewski, Extensions of measures, Fund. Math., 36 (1949), 269-276.

12. J. Mack and D. O. Johnson, The Dedekind completion of $C(X)$, Pacific J. Math.

20 (1967), 231-243.

13. J. Marik, The Baire and Borel measures, Czech. J. Math.. 7 (1957), 248-253.

14. W. Moran, Measures and mappings on topological spaces, Proc. London Math. Soc., 31, 20 (1974), 507-524.

15. - Measures on metacompact spaces, Proc. London Math. Soc., 31, 20 (1974), 507-524.

16. - The additivity of measures on completely regular spaces, J. London Math. Soc., 43 (1968), 633-639.

17. S. E. Mosiman and R. F. Wheeler, The strict topology in a completely regular setting; Relations to topological measure theory, Canad. J. Math., 24 (1972), 73-91.

18. A. Sultan, A general measure extension procedure, Proc. Amer. Math. Soc., 69 No. 1, (1978), 37-45.

19. Measure compactification and representation, Canad. J. Math., 30 No. 1, (1978), 54-65.

20. M. D. Weir, Hewitt-Nachbin Spaces, Mathematics Studies 17, North Holland, Amsterdam.

Received April 6, 1979 and in revised form June 21, 1979.

Polytechnic Institute of New York

BROOKLYN, NY 11201

AND

QueEnS College

Flushing, NY 11367 



\title{
PACIFIC JOURNAL OF MATHEMATICS
}

\section{EDITORS}

\author{
DoNALD BABBITT (Managing Editor) \\ University of California \\ Los Angeles, CA 90024 \\ HUGo RossI \\ University of Utah \\ Salt Lake City, UT 84112 \\ C. C. MOORE and ANDREW OGG \\ University of California \\ Berkeley, CA 94720
}

J. DugundJI

Department of Mathematics

University of Southern California

Los Angeles, CA 90007

R. FINN and J. Milgram

Stanford University

Stanford, CA 94305

\section{ASSOCIATE EDITORS}
E. F. BeCKenbach
B. H. NeumanN
F. WOLF
K. YoshidA

\section{SUPPORTING INSTITUTIONS}

\author{
UNIVERSITY OF BRITISH COLUMBIA \\ CALIFORNIA INSTITUTE OF TECHNOLOGY \\ UNIVERSITY OF CALIFORNIA \\ MONTANA STATE UNIVERSITY \\ UNIVERSITY OF NEVADA, RENO \\ NEW MEXICO STATE UNIVERSITY \\ OREGON STATE UNIVERSITY \\ UNIVERSITY OF OREGON
}

\author{
UNIVERSITY OF SOUTHERN CALIFORNIA \\ STANFORD UNIVERSITY \\ UNIVERSITY OF HAWAII \\ UNIVERSITY OF TOKYO \\ UNIVERSITY OF UTAH \\ WASHINGTON STATE UNIVERSITY \\ UNIVERSITY OF WASHINGTON
}

The Supporting Institutions listed above contribute to the cost of publication of this Journal, but they are not owners or publishers and have no responsibility for its content or policies.

Mathematical papers intended for publication in the Pacific Journal of Mathematics should be in typed form or offset-reproduced, (not dittoed), double spaced with large margins. Please do not use built up fractions in the text of the manuscript. However, you may use them in the displayed equations. Underline Greek letters in red, German in green, and script in blue. The first paragraph or two must be capable of being used separately as a synopsis of the entire paper. Please propose a heading for the odd numbered pages of less than 35 characters. Manuscripts, in triplicate, may be sent to any one of the editors. Please classify according to the scheme of Math. Reviews, Index to Vol. 39. Supply name and address of author to whom proofs should be sent. All other communications should be addressed to the managing editor, or Elaine Barth, University of California, Los Angeles, California, 90024.

50 reprints to each author are provided free for each article, only if page charges have been substantially paid. Additional copies may be obtained at cost in multiples of 50 .

The Pacific Journal of Mathematics is issued monthly as of January 1966. Regular subscription rate: $\$ 84.00$ a year $(6$ Vols., 12 issues). Special rato: $\$ 42.00$ a year to individual members of supporting institutions.

Subscriptions, orders for numbers issued in the last three calendar years, and changes of address shoud be sent to Pacific Journal of Mathematics, P.O. Box 969, Carmel Valley, CA 93924, U.S.A Old back numbers obtainable from Kraus Periodicals Co., Route 100, Millwood, NY 10546.

\section{PUBLISHED BY PACIFIC JOURNAL OF MATHEMATICS, A NON-PROFIT CORPORATION}

Printed at Kokusai Bunken Insatsusha (International Academic Printing Co., Ltd.). 8-8, 3-chome, Takadanobaba, Shinjuku-ku, Tokyo 160, Japan. 


\section{Pacific Journal of Mathematics}

\section{Vol. 86, No. 2 December, 1980}

Graham Donald Allen, David Alan Legg and Joseph Dinneen Ward, Hermitian

liftings in Orlicz sequence spaces ............................... 379

George Bachman and Alan Sultan, On regular extensions of measures ........ 389

Bruce Alan Barnes, Representations Naimark-related to $*$-representations; a

correction: "When is a representation of a Banach $*$-algebra

Naimark-related to a $*$-representation?" ........................ 397

Earl Robert Berkson, One-parameter semigroups of isometries into $H^{p} \ldots \ldots .403$

M. Brodmann, Piecewise catenarian and going between rings ............ 415

Joe Peter Buhler, A note on tamely ramified polynomials ............... 421

William Lee Bynum, Normal structure coefficients for Banach spaces ........ 427

Lung O. Chung, Biharmonic and polyharmonic principal functions ......... 437

Vladimir Drobot and S. McDonald, Approximation properties of polynomials

with bounded integer coefficients .............................

Giora Dula and Elyahu Katz, Recursion formulas for the homology of

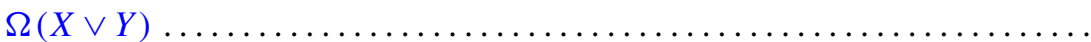

John A. Ernest, The computation of the generalized spectrum of certain Toeplitz operators ...................................... 463

Kenneth R. Goodearl and Thomas Benny Rushing, Direct limit groups and the

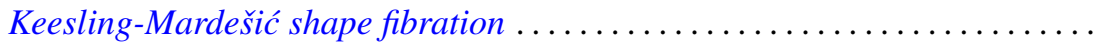

Raymond Heitmann and Stephen Joseph McAdam, Good chains with bad contractions

Patricia Jones and Steve Chong Hong Ligh, Finite hereditary near-ring-semigroups .

Yoshikazu Katayama, Isomorphisms of the Fourier algebras in crossed

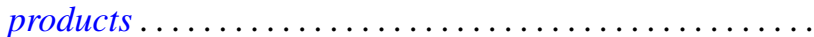

Meir Katchalski and Andrew Chiang-Fung Liu, Symmetric twins and common transversals. .

Mohammad Ahmad Khan, Chain conditions on subgroups of LCA groups ....

Helmut Kröger, Padé approximants on Banach space operator equations ...

Gabriel Michael Miller Obi, An algebraic extension of the Lax-Milgram theorem . .

S. G. Pandit, Differential systems with impulsive perturbation .

Richard Pell, Support point functions and the Loewner variation ...

J. Hyam Rubinstein, Dehn's lemma and handle decompositions of some

4-manifolds

James Eugene Shirey, On the theorem of Helley concerning finite-dimensional subspaces of a dual space.

Oved Shisha, Tchebycheff systems and best partial bases.... 